\title{
Fluid overload and kidney failure in children with severe sepsis and septic shock: A cohort study
}

\author{
Omar E. Naveda Romero, M.D. ${ }^{a}$ and Andrea F. Naveda Meléndez, student ${ }^{a}$
}

\begin{abstract}
Introduction. In children with sepsis, fluid overload as a result of an aggressive fluid replacement or excessive fluid administration may result in kidney impairment and increased mortality.

Objective. To determine the association between fluid overload and the rate of kidney failure in a group of children with severe sepsis and septic shock.

Population and methods. This was a prospective cohort study conducted in the intensive care unit of Hospital Universitario de Pediatría Dr. Agustín Zubillaga (Barquisimeto, Lara State, Venezuela), between March 2013 and May 2016, in children with severe sepsis or septic shock. Results. Onehundredandforty-ninepatientswere included in the analysis. Sepsis predominated in $59.7 \%$ of cases; patients' average age was $6.4 \pm 3.3$ years old, their average weight was $17.8 \pm 3.6 \mathrm{~kg}$, $30.2 \%$ had fluid overload, and overall mortality was $25.5 \%$. Kidney failure occurred in $16.1 \%$ of cases. A binary logistic regression model was used to identify fluid overload (odds ratio [OR]: $1.5 ; 95 \%$ confidence interval $[\mathrm{CI}]: 1.2-4.9, p=0.028)$ and shock for more than 2 days (OR: $1.7 ; 95 \%$ CI: $1.3-6.3, p=0.039)$ as independent predictors of kidney failure. In addition, a significant increase in the risk of mortality among children with kidney failure and fluid overload was observed as per the Kaplan-Meier method ( $p=0.019$ ).

Conclusion. Fluid overload and shock for more than 2 days increase the risk for kidney failure in critically ill children with severe sepsis and septic shock.

Key words: fluid balance, sepsis, shock, kidney failure, mortality.
\end{abstract}

a. Hospital

Universitario de Pediatría Dr. Agustín Zubillaga , Barquisimeto, Lara State, Venezuela.

b. High school graduate. University student. School of Health Sciences, Universidad Centroccidental Lisandro Alvarado. Venezuela.

E-mail address:

Omar E. Naveda

Romero, M.D.: omarnavedamd@ yahoo.com.

Funding:

None.

Conflict of interest: None.

Received: 6-19-2016 Accepted: 9-26-2016 http://dx.doi.org/10.5546/aap.2017.eng.118

To cite: Naveda Romero OE, Naveda Meléndez AF. Fluid overload and kidney failure in children with severe sepsis and septic shock: A cohort study. Arch Argent Pediatr 2017;115(2):118-124.

\section{INTRODUCTION}

The worldwide incidence of acute kidney failure for any cause in hospitalized children has been estimated at 1 every 3 patients, with a $14 \%$ mortality rate. Such rate increases depending on the severity of the underlying disease, and may reach up to $80 \%$ among critically ill patients admitted to the intensive care unit (ICU). ${ }^{1}$ One of the main causes of kidney failure is sepsis; approximately one-third of children with sepsis progress to kidney failure. ${ }^{2}$

At present, intravenous replacement fluids in the initial treatment of sepsis and septic shock are the fundamental pillars of hemodynamic management in these patients; however, it has been questioned that an excessive use of these fluids may cause a harmful effect on the disease course and any resulting complication. The use of an excessive fluid volume may be associated with an impairment in respiratory function, coagulopathy, and increased risk for brain swelling. ${ }^{3}$

There is controversy regarding the effectiveness, nature, scope, and duration of fluid therapy in severe sepsis and septic shock. It is not possible to rule out that a positive fluid balance may be just a marker of the severity of the underlying disease rather than an independent predictor of complications. A high frequency of fluid overload has been reported in the first 24 hours of fluid therapy in critically ill patients with respiratory failure, patients on extracorporeal life support, or patients receiving renal replacement therapy. ${ }^{5}$ The mechanisms involved in the damage caused by fluid overload that may share a common pathway resulting in an increased mortality or systemic complications are still unknown or merely speculative.

The primary objective of this research was to determine the association between fluid overload and the rate of kidney failure in a group of children with severe sepsis and septic shock.

\section{POPULATION AND METHODS}

This was a prospective cohort study conducted in patients 
admitted consecutively to the ICU of Hospital Universitario de Pediatría Dr. Agustín Zubillaga (Barquisimeto, Lara State, Venezuela), between March 2013 and May 2016. Inclusion criteria were children aged 2-13, diagnosed with severe sepsis or septic shock, and who had a communityacquired infection and an initial serum creatinine level of $1.5 \mathrm{mg} / \mathrm{dL}$.

Patients were identified by a specific group of pediatric intensivists at the emergency department, the inpatient ward or the ICU, and were in follow-up until hospital discharge or death. The following patients were excluded: patients with cyanotic congenital heart disease, or community-acquired septic shock, those who received fluid replacement outside the hospital (given that it was not possible to adequately record the exact amount of fluids given before patient admission) or vasopressors before being transferred to the hospital, those who received colloid solutions for fluid resuscitation, had nosocomial sepsis, were exposed to nephrotoxic substances, had burns, surgery, polytrauma, or hematological malignancy, and those who died within 72 hours after ICU admission. A coded database was created to protect data confidentiality. The study was approved by the Bioethics Committee, and an informed consent was obtained.

Severe sepsis and septic shock were defined based on the criteria established by the international guideline development committee for the "Surviving Sepsis Campaign."6 Severe sepsis was defined as an acute infection with organ dysfunction whereas septic shock was defined as multiple organ dysfunction in the presence of an acute infection requiring vasopressors for more than 12 hours. To establish shock duration, the time from vasopressor initial use until withdrawal once the patient was hemodynamically stable was recorded. Kidney failure was defined as a serum creatinine level of $2 \mathrm{mg} / \mathrm{dL}$ or higher, with a urine output of less than $0.5 \mathrm{~mL} / \mathrm{kg} /$ hour over 12 hours, as per the Kidney Disease: Improving Global Outcomes (KDIGO) criteria. ${ }^{7}$

Fluid balance was estimated by subtracting the total amount of fluid output every 24 hours from the total amount of fluids administered both intravenously and orally. Fluid output included any fluid loss besides urine, except imperceptible loss. Fluid overload was established when fluid balance in the first 72 hours of patient admission was more than $10 \%$ of the patient's body weight at the time of admission to the ICU. Only crystalloid solutions were used. The Holliday-Segar method was used for electrolyte management, with volume expansion between 10 and $20 \mathrm{cc} / \mathrm{kg}$ of body weight, deficit correction as per the percentage of dehydration, and sodium, potassium, and calcium maintenance as needed.

Based on cohort studies conducted in children with sepsis and shock, ${ }^{8}$ and for a 31\% kidney failure risk in subjects exposed to fluid overload, a $10 \%$ risk in non-exposed subjects, a 2.3 exposed/ non-exposed ratio, a 95\% confidence level, and an $80 \%$ power, the sample size was estimated at 45 subjects exposed to fluid overload and 104 subjects without exposure, with Yates' correction (overall sample: 149 subjects). The Epidat 3.1 software was used to estimate the sample, and the SPSS Statistics ${ }^{\circledR}$ software, version 17.0, to perform a statistical analysis. The descriptive analysis included mean and standard deviation values for quantitative outcome measures, and percentages for qualitative outcome measures. Pearson's $\chi^{2}$ test or Fisher's test was used to compare qualitative data, and Student's t test or the MannWhitney U test was used for quantitative data, when applicable. A $p$ value $<0.05$ was regarded as significant. Outcome measures compared as per the presence of kidney failure were studied using a univariate analysis and a box plot. Then, using a Mantel-Haenszel analysis, the relationship between kidney failure and fluid overload was stratified based on outcome measures of interest due to potential confounding factors. Confusion was considered present if association measures in each stratum were similar to one another and different from the overall association measure, or when the confounding bias was over $10 \%$. Clinically relevant outcome measures that were associated with kidney failure in the univariate analysis were used to create a logistic regression model with a $p$ value equal to or lower than 0.1 . An "introductory" procedure was used as inclusion criteria. The Hosmer-Lemeshow goodness-of-fit test was performed. Based on the length of stay and overall mortality outcome measures, a Kaplan-Meier survival curve was created, using kidney failure stratified as per the presence of fluid overload as factor; its statistical significance was estimated using a log-rank test.

\section{RESULTS}

A total of 149 critically ill children with severe sepsis or septic shock were included. The average age was $6.4 \pm 3.3$ years old, the average 
weight was $17.8 \pm 3.6 \mathrm{~kg}$, the pediatric risk of mortality (PRISM) was $16 \pm 6$ points, the pediatric logistic organ dysfunction (PELOD) score was $17 \pm 11$ points, and $20.8 \%$ of infections had been caused by Gram-negative microorganisms. The most predominant source of infection was the respiratory tract. The average time to kidney failure was $6.2 \pm 1.3$ days, overall mortality was $25.5 \%$, and the cumulative fluid balance in the first 72 hours since patient admission to the ICU was $1302 \pm 825 \mathrm{~mL}$. Fluid overload was observed in 45 subjects (Table 1 ).

Kidney failure occurred in 24 patients $(16.1 \%)$. Table 2 shows the characteristics of these children compared to those who did not develop kidney failure. The proportion was higher in the group of patients with an abdominal source of infection, $(50.0 \%$ versus $21.6 \%, p=0.004)$, with a

TABLE 1. Characteristics of the study population

\begin{tabular}{|c|c|}
\hline Outcome measures & $\begin{array}{c}\text { All } \\
(\mathrm{N}=149)\end{array}$ \\
\hline \multicolumn{2}{|l|}{ Demographic characteristics $( \pm \mathrm{SD})$} \\
\hline Age (years old) & $6.4 \pm 3.3$ \\
\hline Male sex $(\%)$ & $86(57.7)$ \\
\hline Weight $(\mathrm{kg})$ & $17.8 \pm 3.6$ \\
\hline PRISM (points) & $16 \pm 6$ \\
\hline PELOD (points) & $17 \pm 11$ \\
\hline \multicolumn{2}{|l|}{ Clinical data } \\
\hline Severe sepsis $(\%)$ & $89(59.7)$ \\
\hline Septic shock $(\%)$ & $60(40.3)$ \\
\hline Kidney failure (\%) & $24(16.1)$ \\
\hline Liver failure (\%) & $12(8.1)$ \\
\hline \multicolumn{2}{|l|}{ Microorganisms } \\
\hline Gram-negative (\%) & $31(20.8)$ \\
\hline Gram-positive (\%) & $29(19.5)$ \\
\hline Fungi (\%) & $10(6.7)$ \\
\hline \multicolumn{2}{|l|}{ Source of infection } \\
\hline Respiratory tract (\%) & $54(36.2)$ \\
\hline Abdominal $(\%)$ & $39(26.2)$ \\
\hline Soft tissue $(\%)$ & $24(16.1)$ \\
\hline Urinary tract $(\%)$ & $18(12.1)$ \\
\hline CNS $(\%)$ & $14(9.4)$ \\
\hline \multicolumn{2}{|l|}{ Outcome $( \pm S D)$} \\
\hline Length of stay at the ICU (days) & $8.3 \pm 2.8$ \\
\hline Total length of stay (days) & $16.7 \pm 4.5$ \\
\hline Days passed between hospitalization & \\
\hline and admission to ICU & $0.9 \pm 0.9$ \\
\hline Mechanical ventilation (\%) & $133(89.3)$ \\
\hline Time to kidney failure (days) & $6.2 \pm 1.3$ \\
\hline Mortality in the ICU $(\%)$ & $27(18.1)$ \\
\hline Overall mortality (\%) & $38(25.5)$ \\
\hline Cumulative fluid balance at $72 \mathrm{~h}(\mathrm{~mL})$ & $1302 \pm 825$ \\
\hline Fluid overload $(\%)$ & $45(30.2)$ \\
\hline
\end{tabular}

PRISM: Pediatric Risk of Mortality; PELOD: Pediatric Logistic Organ Dysfunction; CNS: central nervous system; ICU: intensive care unit.

\pm SD: mean \pm standard deviation. higher frequency of kidney failure in the Gramnegative infection group ( $41.7 \%$ versus $16.8 \%$, $p=0.006)$, a longer duration of shock $(2.9 \pm 1.1$ versus $1.9 \pm 1.0$ days, $p=0.008)$, and a higher frequency of shock for more than 2 days $(90.0 \%$ versus $56.0 \%, p=0.044)$. Also in this group, there were differences in fluid balance, which was significantly higher at 48 and 72 hours, and also in the cumulative fluid balance at 72 hours $(1670 \pm 836 \mathrm{~mL}$ versus $1232 \pm 808 \mathrm{~mL}, p=0.017)$, with a higher frequency of patients who had fluid overload in the first 72 hours $(50.0 \%$ versus $26.4 \%$, $p=0.021)$, and a higher overall mortality $(41.7 \%$ versus $22.4 \%, p=0.047$ ).

Figure 1 shows a box plot that describes a significantly greater cumulative fluid level in the group of patients with kidney failure, reflected in the fluid balance at 48 hours $(p=0.013)$, at 72 hours $(p=0.039)$, and the cumulative fluid balance at 72 hours $(p=0.017)$. A multivariate analysis using a binary logistic regression was done subsequently (Table 3), considering potentially predictive outcome measures (independent outcome measures), which showed a $p$ value equal to or lower than 0.1 in the univariate analysis. Once the logistic regression model was adjusted for kidney failure as output variable (dependent outcome measure), it was observed that fluid overload (odds ratio [OR]: $1.5 ; 95 \%$ confidence interval [CI]: 1.2-4.9, $p=0.028$ ) and shock for more than 2 days (OR: 1.7; 95\% CI: 1.36.3, $p=0.039$ ) were independent predictors associated with the development of kidney failure in children with severe sepsis. The regression did not show an association with age between 7 and 13 years old, a PELOD score $>20$ points, an abdominal source of infection, or a Gram-negative infection.

Figure 2 shows the Kaplan-Meier survival curve as per the presence of kidney failure stratified by fluid overload. The curve describes a significant increase in the mortality risk among children with kidney failure in the "patients with fluid overload" stratum. A significant difference $(p=0.019)$ was observed in the log-rank test (Mantel-Cox). The "patients without fluid overload" stratum did not show a significant difference in cumulative survival among patients with or without kidney failure $(p=0.330)$.

\section{DISCUSSION}

The potential association between fluid overload within 72 hours after admission to the ICU in a cohort of critically ill children with 
severe sepsis or septic shock and an adverse outcome, kidney failure, was explored. Results were essentially similar to those described in recent reports, which suggest an independent association between a positive fluid balance in the first days of admission to the ICU and an

TABLE 2. Univariate analysis as per the presence of kidney failure

\begin{tabular}{|c|c|c|c|}
\hline \multirow[t]{2}{*}{ Outcome measure* } & \multicolumn{2}{|c|}{ Kidney failure } & \multirow[t]{2}{*}{$p^{* *}$} \\
\hline & Yes $(n=24)$ & No $(n=125)$ & \\
\hline \multicolumn{4}{|l|}{ Demographic characteristics } \\
\hline Age (years old, $\mathrm{X} \pm \mathrm{SD}$ ) & $5.5 \pm 3.7$ & $6.5 \pm 3.2$ & 0.144 \\
\hline $7-13$ years old $(\%)$ & $13(54.2)$ & $91(72.8)$ & 0.069 \\
\hline Male sex $(\%)$ & $17(70.8)$ & $69(55.2)$ & 0.156 \\
\hline Weight $(\mathrm{kg} ; \mathrm{X} \pm \mathrm{SD})$ & $17.4 \pm 4.9$ & $17.9 \pm 3.3$ & 0.587 \\
\hline PRISM (points; $X \pm S D$ ) & $15 \pm 6$ & $16 \pm 5$ & 0.464 \\
\hline PELOD (points; $X \pm S D$ ) & $17 \pm 14$ & $17 \pm 11$ & 0.890 \\
\hline PELOD $>20$ points $(\%)$ & $5(20.8)$ & $48(38.4)$ & 0.100 \\
\hline \multicolumn{4}{|l|}{ Source of infection } \\
\hline CNS (\%) & $2(8.3)$ & $12(9.6)$ & 0.846 \\
\hline Respiratory tract (\%) & $6(25.0)$ & $48(38.4)$ & 0.211 \\
\hline Abdominal (\%) & $12(50.0)$ & $27(21.6)$ & 0.004 \\
\hline Soft tissue $(\%)$ & $3(12.5)$ & $21(16.8)$ & 0.600 \\
\hline Urinary tract $(\%)$ & $1(4.2)$ & $17(13.6)$ & 0.194 \\
\hline \multicolumn{4}{|l|}{ Microorganisms } \\
\hline Gram-positive (\%) & $2(8.3)$ & $27(21.6)$ & 0.133 \\
\hline Gram-negative (\%) & $10(41.7)$ & $21(16.8)$ & 0.006 \\
\hline Fungi (\%) & $1(4.2)$ & $9(7.2)$ & 0.586 \\
\hline \multicolumn{4}{|l|}{ Clinical data } \\
\hline Septic shock $(\%)$ & $10(41.7)$ & $50(40.0)$ & 0.879 \\
\hline Duration of shock (days; $X \pm S D$ ) & $2.9 \pm 1.1$ & $1.9 \pm 1.0$ & 0.008 \\
\hline Shock for more than 2 days ${ }^{\S}(\%)$ & $9(90.0)$ & $28(56.0)$ & 0.044 \\
\hline Liver failure $(\%)$ & $3(12.5)$ & $9(7.2)$ & 0.382 \\
\hline \multicolumn{4}{|l|}{ Outcome } \\
\hline Mechanical ventilation (\%) & $22(91.7)$ & $111(88.8)$ & 0.678 \\
\hline Days on mechanical ventilation $(X \pm S D)$ & $3.8 \pm 2.8$ & $3.8 \pm 2.3$ & 0.998 \\
\hline Mechanical ventilation for 5 or more days ${ }^{\ddagger}(\%)$ & $8(36.4)$ & $44(39.6)$ & 0.774 \\
\hline \multicolumn{4}{|l|}{ Fluid balance } \\
\hline 24 hours $(\mathrm{mL} ; \mathrm{X} \pm \mathrm{SD})$ & $434 \pm 200$ & $339 \pm 261$ & 0.089 \\
\hline 48 hours $(m L ; X \pm S D)$ & $591 \pm 282$ & $432 \pm 285$ & 0.013 \\
\hline 72 hours $(\mathrm{mL} ; \mathrm{X} \pm \mathrm{SD})$ & $634 \pm 373$ & $462 \pm 369$ & 0.039 \\
\hline Cumulative fluid balance at 72 hours $(X \pm S D)$ & $1670 \pm 836$ & $1232 \pm 808$ & 0.017 \\
\hline Fluid overload (\%) & $12(50.0)$ & $33(26.4)$ & 0.021 \\
\hline Overall mortality $(\%)$ & $10(41.7)$ & $28(22.4)$ & 0.047 \\
\hline
\end{tabular}

PRISM: Pediatric Risk of Mortality; PELOD: Pediatric Logistic Organ Dysfunction; CNS: central nervous system; $\mathrm{X} \pm \mathrm{SD}$ : mean and standard deviation.

** Pearson's $\chi^{2}$ test or Fisher's test for qualitative data, and Student's $t$ test or Mann-Whitney U test for quantitative data. $\S$ Estimated only in the septic shock group.

$¥$ Estimated only in the mechanical ventilation group.

TABLE 3. Multivariate logistic regression for kidney failure risk $(N=149)$

\begin{tabular}{lcccc}
\hline Outcome measure & $\beta$ & OR & 95\% CI & $p$ \\
\hline Age 7-13 years old & -0.948 & 0.9 & $0.8-2.5$ & 0.095 \\
PELOD > 20 points & 2.184 & 0.8 & $0.5-7.3$ & 0.145 \\
Abdominal source of infection & -1.588 & 0.5 & $0.2-2.1$ & 0.107 \\
Gram-negative infection & -0.357 & 0.9 & $0.1-5.2$ & 0.704 \\
Shock for more than 2 days & -1.982 & 1.7 & $1.3-6.3$ & 0.039 \\
Fluid overload & -1.503 & 1.5 & $1.2-4.9$ & 0.028 \\
\hline
\end{tabular}

$\beta$ : beta coefficient; OR: odds ratio; CI: confidence interval. PELOD: Pediatric Logistic Organ Dysfunction.

The model correctly classified $83.9 \%$ of cases.

Hosmer-Lemeshow test $=0.447$. 
unfavorable outcome, including sepsis-induced kidney failure.

There is clear evidence that the early administration of fluids for a specific hemodynamic purpose in patients with sepsis and septic shock is associated with a

FIGURE 1. Box plot for the different fluid balance levels as per the presence of kidney failure

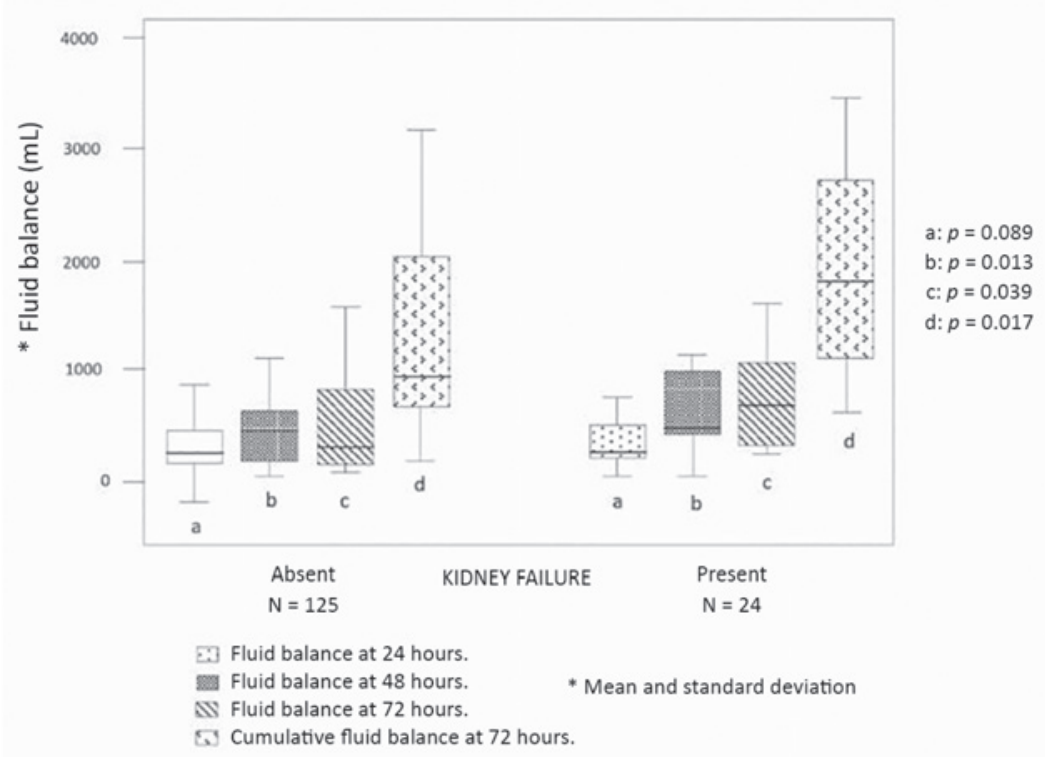

FIgURE 2. Kaplan-Meier survival curve
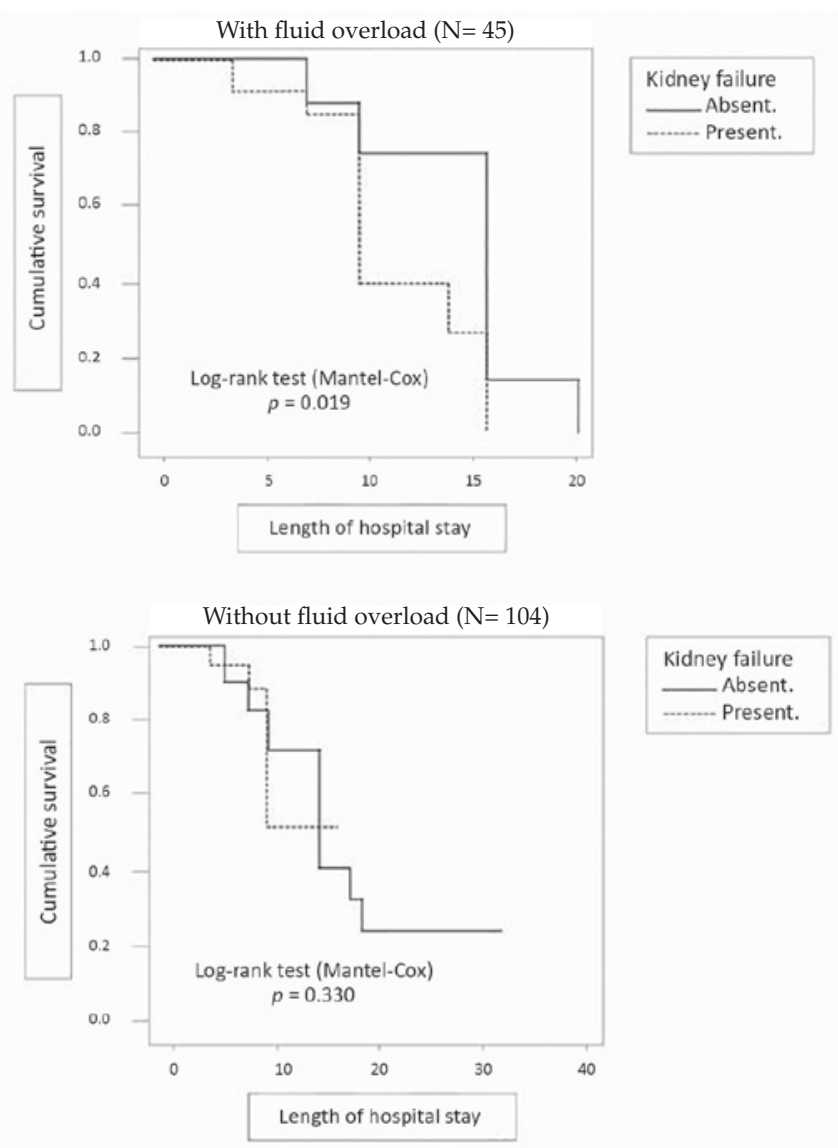
significant improvement in survival at 28 and 60 days following admission. ${ }^{9}$ Contradictory to these findings, fluid overload is described as an independent predictor of adverse events in critically ill children with severe sepsis and shock. ${ }^{10}$ In addition, some investigators have observed that fluid overload remained independently associated with adverse outcomes, such as kidney failure, once all confounding factors for disease severity and hemodynamic instability had been ruled out. ${ }^{11}$

The pathophysiology of sepsis-induced kidney failure may be more complex than what has been previously described. Hypovolemia, heart failure, and hypoperfusion may be the consequence of shock; therefore, kidney failure is attributed to ischemia and hemodynamic changes. However, kidney failure is not the result of hypoperfusion only; it may also be caused by the action of inflammatory cascade mediators or be developed with normal blood flow, and even increase with fluid overload. ${ }^{12}$

The specific mechanisms whereby this cumulative positive fluid balance influences on prognosis remain unknown or are merely speculative, just like the association with kidney failure. Such common acute kidney dysfunction in a critically ill patient and severe complication in the course of septic shock may worsen prognosis. ${ }^{13}$ In this cohort, the association between fluid overload and kidney failure was observed in the univariate analysis whereas fluid overload was found to be an independent predictor of kidney failure in the multivariate analysis.

The association between fluid overload and kidney failure may be explained by several pathophysiological, although speculative, pathways: fluid overload would cause peripheral and visceral edema, result in an impaired functioning of certain organs, e.g. the kidney, and be associated with multiorgan failure; antibiotic hydrophilic dilution would reduce antibiotic effectiveness and let the infection progress and interact with proinflammatory and anti-inflammatory factors; serum creatinine hemodilution would delay the diagnosis of acute kidney damage. ${ }^{14}$ In addition, the kidney is an encapsulated organ with limited capacity to accommodate excess fluids; therefore, there is increased renal subcapsular pressure, which may result in an increased interstitial fluid pressure with major consequences on regional blood flow and functional impairment. ${ }^{15}$ Increased intra- abdominal pressure, central venous pressure, and renal venous pressure in fluid overload conditions may substantially contribute to a reduced glomerular filtration rate. ${ }^{16}$

Another aspect to be taken into account is that the association between kidney failure and fluid overload has a significant impact on mortality. Hypervolemia and hyperosmolarity may exacerbate capillary leakage in patients with septic shock, which results in a generalized edema, increased intra-abdominal pressure, and pulmonary edema. Such cumulative fluid balance is associated with the development of systemic and regional hypoperfusion, and subsequently, multiorgan failure and an increased mortality risk. ${ }^{17}$ This is consistent with the findings made in this cohort: in the presence of fluid overload, the mortality curve was significantly increased for patients with kidney failure. However, when there was no fluid overload, mortality was similar between patients with and without kidney failure.

Another relevant aspect to be taken into consideration is the time since shock onset. Once secondary hypotension is prolonged over time, it causes a severe reduction in tissue perfusion with reduced oxygen supply, inflammatory mediator alteration, endothelial dysfunction, and cardiac output alteration. ${ }^{18}$ In this case, the administration of fluids and vasoactive agents works in a dynamic manner and may change depending on the patient's course. This situation has also been observed in our study cohort, where the presence of shock for more than 2 days was observed to be an independent predictor of kidney failure in the multivariate regression.

The recommendations for fluid replacement in patients with severe sepsis or septic shock have been standardized in recent years. These recommendations are based on expert opinion or supported by weak physiological data and limited experimental evidence. In spite of these limitations, they are considered the cornerstone of care for sepsis patients. This paradigm is threatened by recent results, which show that a more positive fluid balance, both at the beginning of replacement and the cumulative volume over the following days, is associated with a higher mortality risk among adults and children with severe sepsis or septic shock. ${ }^{19}$ In addition, the association between fluid overload and kidney failure in children with sepsis does not seem to be related to the patient's initial severe condition given that the PRISM and PELOD scores within the first 72 hours of admission to the ICU did not show significant 
differences between the kidney failure group and the group without kidney failure.

Not all reports support the association between fluid overload and kidney failure. Some studies failed to establish a positive fluid balance in the first hours of care at the ICU as an independent predictor of kidney failure. ${ }^{20}$ Such discrepancy may be the result of the heterogeneity of the studied group and the low rate of kidney failure detected in the group who had fluid overload in these studies.

The results observed in this study pose major limitations: an observational, non-interventional design and a cohort with a small number of studied subjects in spite of the adequate sample size estimation. These factors limit the possibility of establishing a strong causal relationship between fluid overload and kidney failure.

It is also worth noting that fluid requirements and the interpretation of fluid balance volumes vary depending on the patient's age. In this study, no stratified analysis was made based on age, and the age range was 2 to 13 years old. The amount of fluids given over the length of stay, both in the hemodynamic instability phase and the subsequent follow-up, was not stratified either. In addition, there is no rigid protocol for the administration of fluids to this type of patients at the place where the study was conducted. Also, using serum creatinine to define kidney failure in critically ill patients is a major limitation because there are more specific markers available.

Finally, the following factors should also be taken into consideration: type of fluid used, administration volume and time, antimicrobial therapy, management of the primary source of infection, serum albumin levels, and use of blood products and diuretics.

Therefore, the cumulative positive fluid balance within the first 72 hours of admission to the ICU may be a risk factor for kidney failure in critically ill patients with sepsis and septic shock. It is also a parameter for serial measurements which may provide important information.

\section{CONCLUSION}

Fluid overload and shock for more than 2 days in critically ill children with sepsis and septic shock are independently associated with kidney failure.

\section{REFERENCES}

1. Antón Gamero M, Fernández Escribano A. Daño renal agudo. Protoc Diagn Ter Pediatr 2014;1:355-71.
2. Basu RK, Chawla LS, Wheeler DS, Goldstein SL. Renal angina: an emerging paradigm to identify children at risk for acute kidney injury. Pediatr Nephrol 2012;27(7):1067-78.

3. Valentine SL, Sapru A, Higgerson RA, Spinella PC, et al. Fluid balance in critically ill children with acutelung injury. Crit Care Med 2012;40(10):2883-9.

4. De La Puente-Diaz de Leon VM, Rivero-Sigarroa E, Domiguez-Cherit G, Namendys-Silva SA. Fluid therapy in severe sepsis and septic shock. Crit Care Med 2013;41(12):e484-5.

5. Prowle JR, Echeverri JE, Ligabo EV, Ronco C, et al. Fluid balance and acute kidney injury. Nat Rev Nephrol 2010;6(2):107-15.

6. Dellinger RP, Levy MM, Rhodes A, Annane D, et al. Surviving Sepsis Campaign: international guidelines for management of severe sepsis and septic shock: 2012. Crit Care Med 2013;41(2):580-637.

7. KDIGO clinical practice guideline for acute kidney injury. Kidney International Supplements 2012;2(1). [Accessed on: September 27 $\left.7^{\text {th }}, 2016\right]$. Available at: http://www. kdigo.org/clinical_practice_guidelines/pdf/KDIGO $\% 20$ AKI\%20Guideline.pdf.

8. Bhaskar P, Dhar AV, Thompson M, Quigley R, et al. Early fluid accumulation in children with shock and ICU mortality: a matched case-control study. Intensive Care Med 2015;41(8):1445-53.

9. Rivers E, Nguyen B, Havstad S, Ressler J, et al. Early goaldirected therapy in the treatment of severe sepsis and septic shock. N Engl J Med 2001;345(19):1368-77.

10. Arikan AA, Zappitelli M, Goldstein SL, Naipaul A, et al. Fluid overload is associated with impaired oxygenation and morbidity in critically ill children. Pediatr Crit Care Med 2012;13(3):253-8.

11. Basu RK, Andrews A, Krawczeski C, Manning P, et al. Acute kidney injury based on corrected serum creatinine is associated with increased morbidity in children following the arterial switch operation. Pediatr Crit Care Med 2013;14(5):e218-24.

12. Gomez H, Ince C, De Backer D, Pickkers P, et al. A unified theory of sepsis-induced acute kidney injury: inflammation, microcirculatory dysfunction, bioenergetics, and the tubular cell adaptation to injury. Shock 2014;41(1):3-11.

13. Ronco C, Kellum JA, Bellomo R, House AA. Potential interventions in sepsis-related acute kidney injury. Clin J Am Soc Nephrol 2008;3(2):531-44.

14. Ávila MO, Rocha PN, Zanetta DM, Yu L, etal. Water balance, acute kidney injury and mortality of intensive care unit patients. J Bras Nefrol 2014;36(3):379-88.

15. Grams ME, Estrella MM, Coresh J, Brower RG, et al. Fluid balance, diuretic use, and mortality in acute kidney injury. Clin J Am Soc Nephrol 2011;6(5):966-73.

16. Ramajaya IB, Wati DK, Gede IB. Association of fluid overload with mortality in pediatric intensive care unit. Crit Care Shock 2016;19(1):8-13.

17. Myburgh JA. Fluid resuscitation in acute illness-time to reappraise the basics. N Engl J Med 2011;364(26):2543-4.

18. MicekST,McEvoy C,McKenzie M,Hampton N, et al. Fluid balance and cardiac function in septic shock as predictors of hospital mortality. Crit Care 2013;17(5):R246.

19. Vaara ST, Korhonen AM, Kaukonen KM, Nisula S, et al. Fluid overload is associated with an increased risk for 90day mortality in critically ill patients with renal replacement therapy: data from the prospective FINNAKI study. Crit Care 2012;16(5):R197.

20. Zhang L, Chen Z, Diao Y, Yang Y, etal. Associations of fluid overload with mortality and kidney recovery in patients with acute kidney injury: a systematic review and metaanalysis. J Crit Care 2015;30(4):860.e7-13. 